\title{
THE MECHANICS OF THE KNEE AND PROSTHESIS DESIGN*
}

\author{
JOHN GOODFELLOW, JOHN O'CONNOR
}

From the Nuffield Orthopaedic Centre and the Department of Engineering Science. Oxford

The mechanisms controlling and limiting movement and serving to transmit load between the femur and the tibia are discussed. Having accounted for the transmission of all components of force and couple across the joint and noted the load-bearing role of the menisci, some principles which might guide the design of knee prostheses are deduced. It is shown that current designs transgress some of these principles. An experimental prosthesis is then described, which incorporates analogues of the natural menisci. The possible practical application of this novel principle has been studied in cadaveric human joints and in living patients.

They hung her from the ceiling,

Yes, they hung up Miss Gee;

And a couple of Oxford Groupers

Carefully dissected her knee

W. H. Auden

Anatomical descriptions of animal joints are customarily limited to the capsule of the joint and its contents. From a functional viewpoint, this custom is unsatisfactory and it is more useful to regard the animal limb as a chain of rigid bars joined together by soft tissues, which include the muscles and their tendons no less than the ligaments. A function of all elements in a skeleton is to transmit load, and we describe the specialised structures of the human knee and its muscles in these terms.

Load transmission. All loads can be expressed in terms of forces acting in three directions at right angles to each other and couples acting about axes in those three directions. For the knee, it is convenient to choose the tibial axis and two axes perpendicular to it in the anteroposterior and mediolateral directions (Fig. 1) since it is customary to describe movements at the joint with respect to these three directions.

Thus, a force in the direction of the tibial axis resists interpenetration of the bones if compressive, distraction of the bones if tensile. Forces in the anteroposterior and mediolateral directions resist (or induce) relative translations of the bones in those directions respectively. A couple about the mediolateral axis resists (or induces) flexion or extension. A couple about the anteroposterior axis resists (or induces) abduction or adduction. And lastly, a couple about the tibial axis resists (or induces) medial and lateral rotation. This convention describes not only all possible movements at the joint but all possible loads to which the limb is subject.

We must distinguish between the mode of transmission of loads along the shafts of the tubular bones and the mode of their transmission from one bone to another (Fig. 2). Within and along the diaphyses, all six

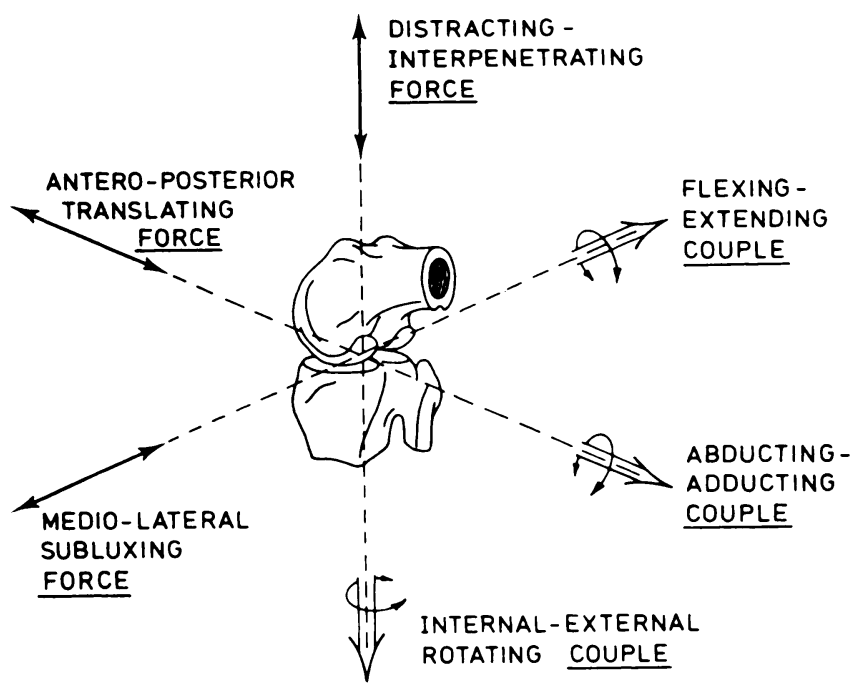

Fig. 1

Reference directions for the knee.

components of load are transmitted by means of tensile, compressive and shear stresses, continuously distributed throughout the bony material. The tubulated form reflects the function of diaphysial bone. By contrast, at the joint, all these six components are transmitted from one bone to the other in some combination of pressure at the articular surfaces and tension in the soft tissues

*Based in part on lectures given to the Sixth Combined Meeting, London, 1976 and to the Twenty-third Meeting of the Orthopaedic Research Society, Las Vegas, 1977.

John Goodfellow, F.R.C.S., Nuffield Orthopaedic Centre, Headington, Oxford OX3 7LD, England.

John O'Connor, B.E., M.A., Ph.D., Department of Engineering Science, University of Oxford, Parks Road, Oxford OX1 3PJ, England. Requests for reprints should be sent to Mr John Goodfellow. 
spanning the joint. The articular surfaces of synovial joints are so smooth and well lubricated as to offer minimal resistance to sliding movements and can therefore transmit no significant shear stress one to another. They do not adhere and cannot therefore transmit tensile stress. They can transmit only compressive stress (pressure) normal to their surfaces. The soft tissues can transmit only tension in the line of their fibres.

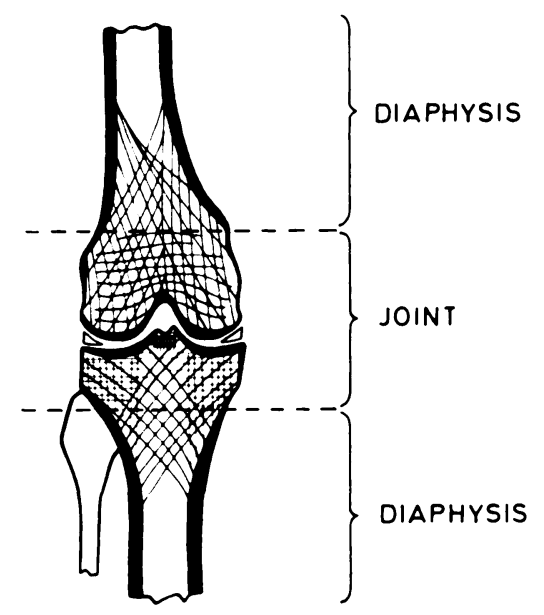

Fig. 2

Tubular and juxta-articular bone. The joint consists of all the material between the dotted lines.

Consequently, the expanded juxta-articular bone at the ends of the shafts and embraced by the ligaments is subjected predominantly to compression, applied to it by the articulating surfaces which it supports. We assume that the trabeculated form of the juxta-articular bone reflects its function and distinguishes it from the tubulated bone which lies beyond the ligamentous insertions. The distinction suggests that different considerations may apply to the design of prostheses which use intramedullary stems to gain attachment to the tubulated bone on the one hand, and those which are attached directly to the trabecular bone on the other. Mobility and stability. Mobility at a joint is conferred by the provision of low-friction bearing surfaces between the bones. Stability is a measure of the degree to which relative movement at the bearing surfaces is limited or resisted.

We should distinguish between passive stability, a measure of the limitation imposed by the length of the ligaments and the contour of the joint surfaces, and active stability when the forces of gravity, ground reaction and muscle action are added. The range of movement in activity cannot lie beyond the range allowed passively.

In most joints, and particularly those of the lower limb, stability is of greater functional importance than mobility and it is possible to describe the natural knee in terms of the mechanisms that resist movement and transmit load. Our purpose is to explain what may appear to be a paradox: that a condylar replacement prosthesis may best confer stability upon the living joint if it is itself completely unstable. We will show that, since natural articular surfaces contribute to joint stability merely by resisting interpenetration of the bones, the only function required of prosthetic surfaces is to do the same.

We shall first describe the forces and couples required to control or limit each of the six possible movements at the knee, in each case distinguishing between active and passive stability. Since force is required either to initiate or to limit movement, the mechanisms that control movement are the same as those used for the transmission of load. The ligaments and tendons that span the joint are favourably oriented to resist distraction of the bones and to develop the tensile component of the couples needed to resist flexion or extension and abduction or adduction. They are less favourably oriented to resist those movements that involve sliding of the articular surfaces-rotation about the tibial axis and translation of the bones in the mediolateral and anteroposterior directions.

These sliding movements are not resisted until the soft tissues tighten and, as they tighten, pull the articular surfaces together (Fig. 3). A balance is reached and further movement is resisted when the component of the soft tissue tension perpendicular to the articular surfaces equals the compressive force on the condyles and the component parallel to the articular surfaces equals the applied shearing force. The range of sliding movement
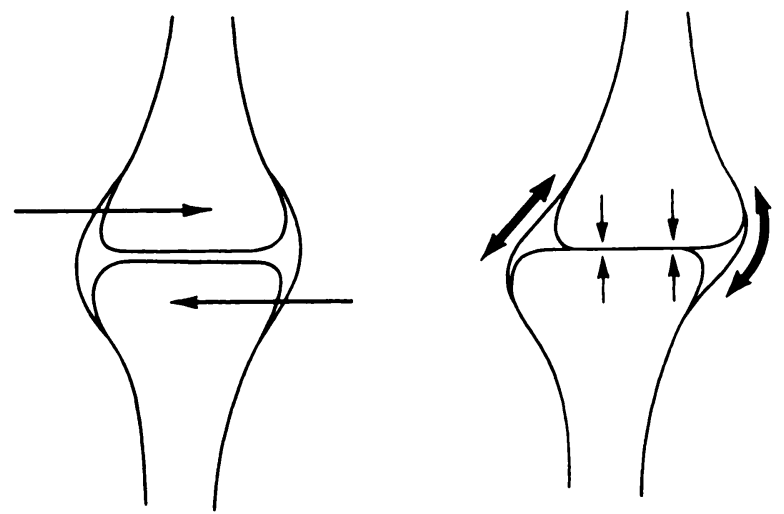

Fig. 3

General method of limiting sliding movements.

available in any joint, therefore, depends on the rate at which the soft tissues tighten in response to a translation or rotation of the bones which, in turn, depends on a complex relationship between the geometry of the articular surfaces and the disposition of the soft tissues.

These generalisations apply to the control of all translatory movements. It is convenient to consider flexion and extension with anteroposterior translation because the former movements occur naturally with the 

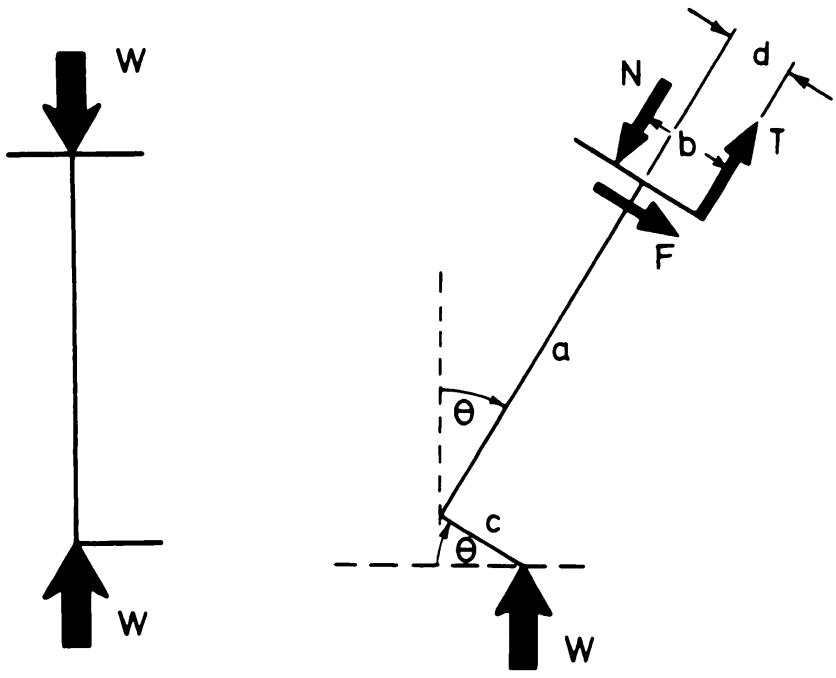

Fig. 4

Equilibrium of the tibia. When vertical, a compressive force at the knee can keep the tibia in equilibrium. When inclined to the vertical, tensile, compressive and shear forces are required. From statics:

$N=W\left[\frac{a}{b} \sin \theta-\left(\frac{c-d}{b}\right) \cos \theta\right] ; T=W\left[\frac{a}{b} \sin \theta-\left(1+\frac{c-d}{b}\right) \cos \theta\right] ;$
$F=W \sin \theta$

The factor $\frac{a}{b}$ in these equations has a value of at least 8 .

latter. We will treat abduction and adduction with mediolateral translations for the same reason.

Flexion and extension with anteroposterior translation. Of all the ways in which the femur and the tibia might move relative to one another, only flexion from the anatomical position is free of passive limitation.

Flexion from any position is resisted actively by the couple of tension in the patellar tendon and compression at the contact areas between the two bones. This couple acts about a lever arm $b$ (Fig. 4), the distance between the line of action of the quadriceps at the insertion of its tendon and the centre of pressure of the contact forces. The large compressive forces described in the caption arise because couples acting through generally short lever arms along the joint surface must resist couples acting through lever arms which can be as long as the shafts of the bones.

In all positions except full extension, the normal knee allows a limited range of anteroposterior gliding of its articular surfaces.

Kapandji (1970) and Huson (1974) have shown that, mainly because of the crossed form of the cruciate ligaments, flexion of the knee is accompanied not only by a sliding movement of the femoral condyles upon the tibia but also by an obligatory rolling movement which carries the contact areas backwards on the tibia in flexion and forwards in extension. The two-dimensional model in Figures 5, 6 and 7, in which the components are held together by crossed wires to simulate the cruciate ligaments, demonstrates that the two bones and the two ligaments constitute a four-bar linkage which commands such movement. Passive stability in the anteroposterior direction is mainly dependent upon the integrity of the cruciates because their fibres lie most nearly in the direction of such movements. Kapandji (1970) has also shown that the collateral ligaments have a similar crossed form when viewed from the side, and they, too, contribute to the mechanism.

The contact areas of a cadaveric knee in extension and flexion are shown in Figure 8. Their obligatory excursion is of the order 0.8 to 1.2 centimetres. Reference to the caption of Figure 4 will show that this backward movement of the contact areas in flexion maximises the power of the quadriceps to extend the

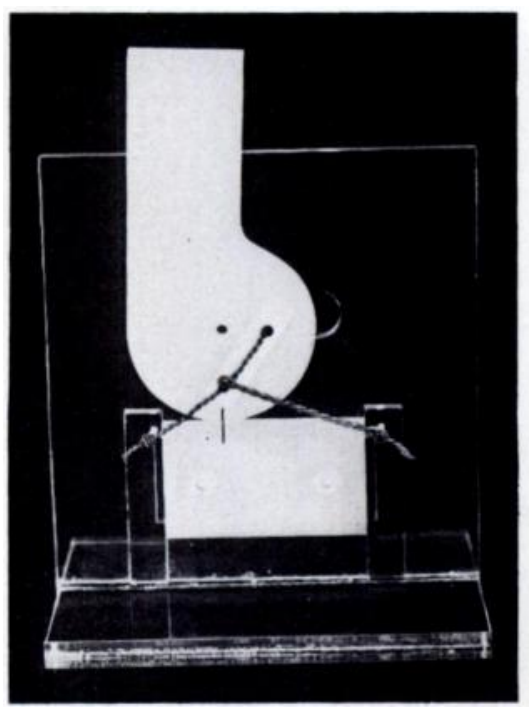

Fig. 5

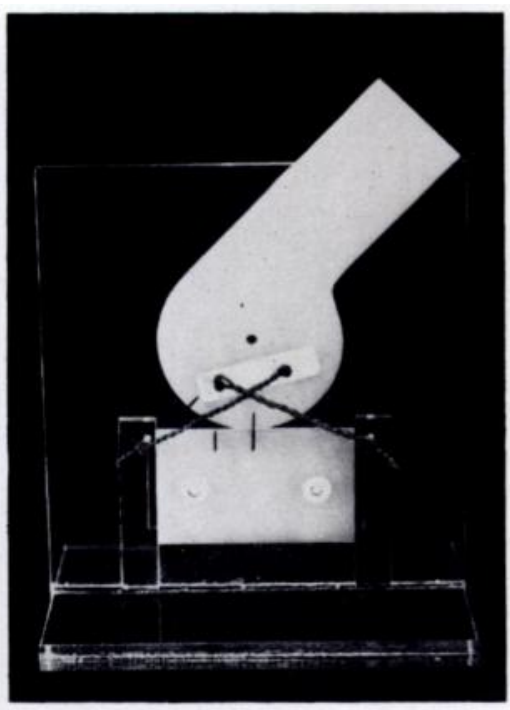

Fig. 6

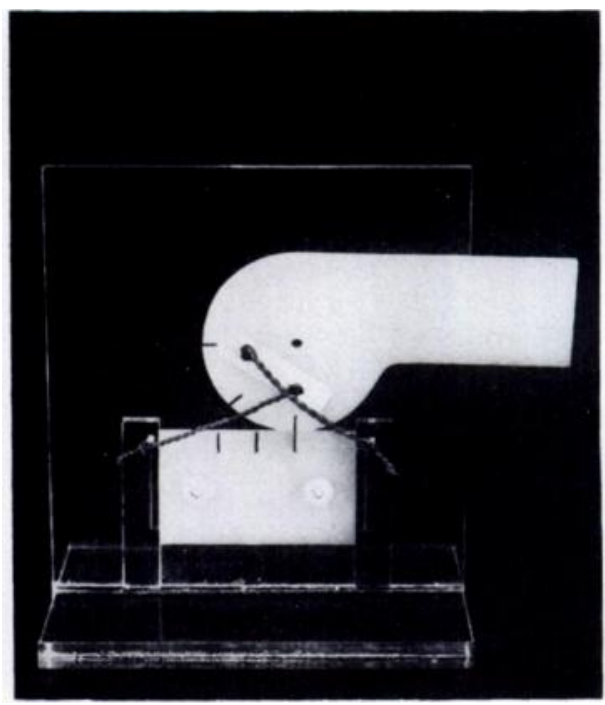

Fig. 7

Figures 5,6 and 7-Two-dimensional model. Marks on the model bones show that the four-bar linkage commands posterior rolling of the femur on the tibia while flexing. The discrepant distances between successive points of contact indicate that posterior rolling is accompanied by anterior sliding in a ratio of about two to one. 
knee (or to resist flexion) and minimises the compressive force at the articular surfaces by making the lever $\operatorname{arm} b$ as large as possible.

Within the narrow range of uncertainty allowed by the ligaments, active control of the position of the a similar state develops even when the natural condyles are replaced by perfectly spherical components, provided that the contact areas can move forward in extension. It is the combination of anterior location of the contact areas and posterior location of the tension

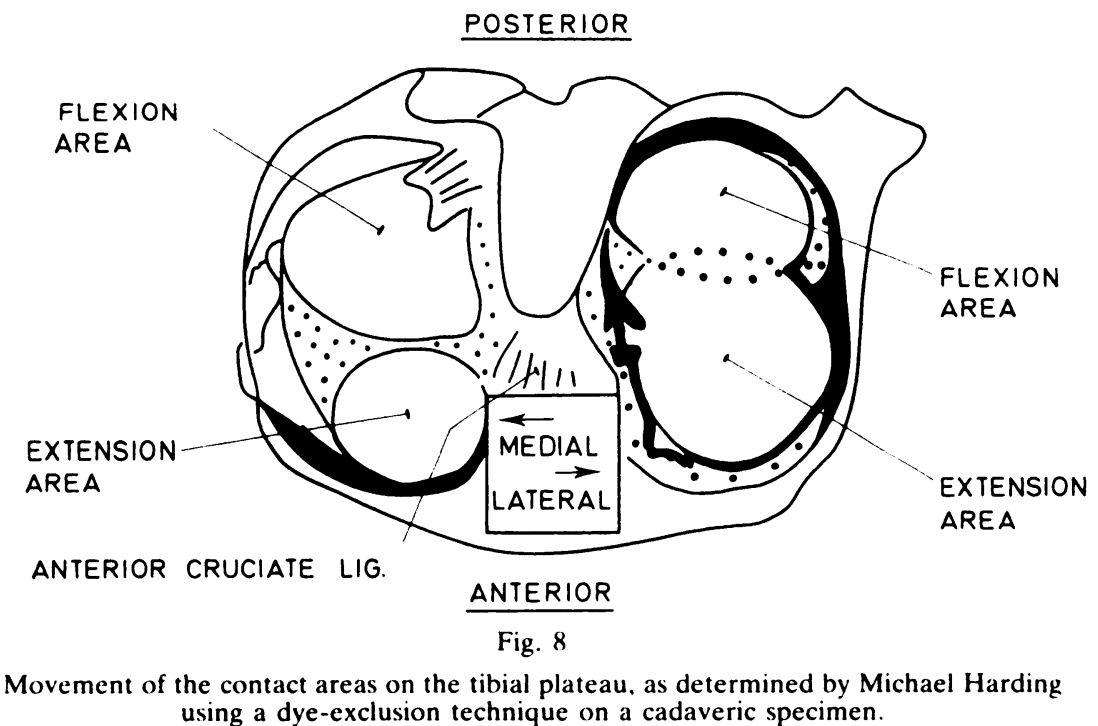

contact areas depends, at any moment, on the balance of the relevant muscle forces. For example, when the quadriceps muscle contracts, the patellofemoral component of its action tends to push the femur backwards on the tibia while the posterior component of the hamstring action tends to pull the tibia backwards on the femur.

Passive limitation of extension depends on the action of a couple of compression at the tibiofemoral contact areas and tension in the soft tissues. It is common practice to assign specific roles to specific ligaments in producing this couple, but it is probably more accurate to say that tension in any of the longitudinally disposed ligaments lying posterior to the centre of pressure of the contact forces must contribute. The posterior capsular ligament clearly enjoys a mechanical advantage over the other ligamentous structures.

Active limitation of extension derives from the flexor muscles which employ a lever arm similar to that of the posterior capsular ligament. In both cases the length of the lever arm is maximised by the forward location of the contact areas ordained by the cruciate mechanism. In Figure 9, both the posterior capsular and the anterior cruciate ligaments are seen to be tight at the passive limit of extension. In the absence of external forces, the two cruciates act together to locate the contact areas, but it is the anterior ligament that mainly resists forces tending to move them backwards on the tibia.

It is commonly supposed that it is the greater radius of curvature of the anterior parts of the femoral condyles which accounts for the tightening of the ligaments and the "locked" state of the fully extended knee. However, elements which allows the development of a couple capable of resisting hyperextension.

Rotation. In all positions short of full extension, a range of rotational freedom is available. It has been measured by Markolf, Mensch and Amstutz (1976) in the

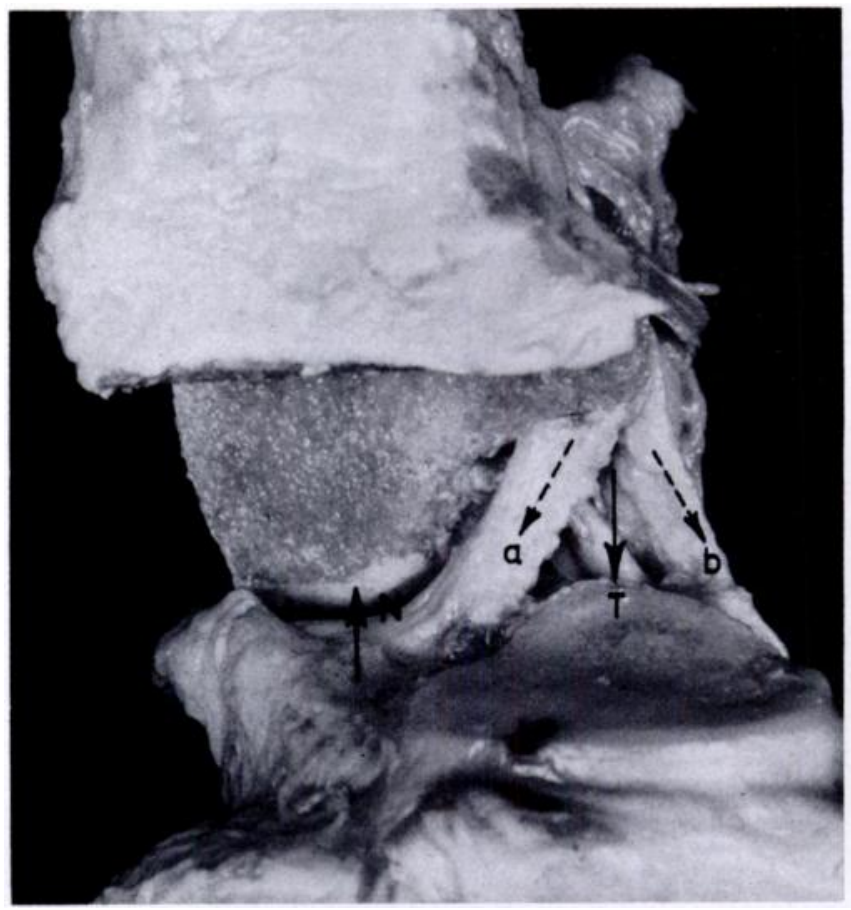

Fig. 9

Specimen with medial femoral condyle removed. Anterior cruciate ligament $(a)$ and posterior capsular ligament $(b)$ both tight at passive limit of full extension. Note anterior location of the contact area between the bones. The resultant $T$ of the tensions in the ligaments and the compressive force $N$ provide the couple which limits extension. 
minimally loaded joint and found to be about 30 degrees with the joint flexed to a right angle.

Several authors have attributed particular responsibilities in resisting medial and lateral rotation to particular ligaments. However, all the tension-bearing elements that span the joint are capable, when they are rendered tight, of contributing to the couples that resist rotation. For example, the circumferential components of the tension forces in all the capsular structures may provide such a couple.

The following observation can readily be made on the living subject. The normal knee, flexed to 90 degrees, is first examined for rotation with the quadriceps relaxed and next with the subject attempting to straighten the joint against the examiner's resistance. The range of rotation will be found to decrease from about 30 degrees to a few degrees only. Having grasped the fact that any soft tissue structure that spans the joint can resist rotation if rendered tight, we can better understand why the fully extended joint will not rotate at all. By definition, the joint which will not further extend is one in which the soft tissues posteriorly will not further lengthen. Since they must lengthen to allow rotation, none can occur.
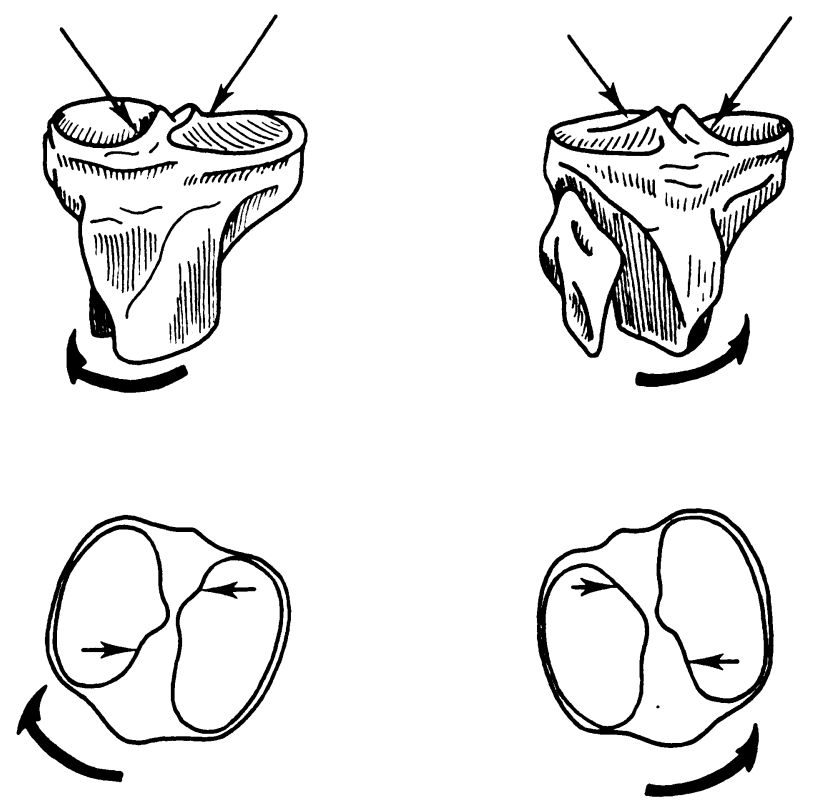

Fig. 10

Contribution of the tibial eminence to the limitation of rotation. In lateral rotation, the posterior location medially and the anterior location laterally of the contact forces provide the lever arm of a couple to balance the applied torque. Vice versa in medial rotation.

The presence of the tibial eminence may give rise to a subsidiary mechanism in limiting rotation (Fig. 10). During rotation, the contact areas move on the tibia, one anteriorly, the other posteriorly. Any tendency of the femur to mount the tibial eminence would be resisted by tension forces in the soft tissues spanning the joint, tension which would be balanced by compression on the

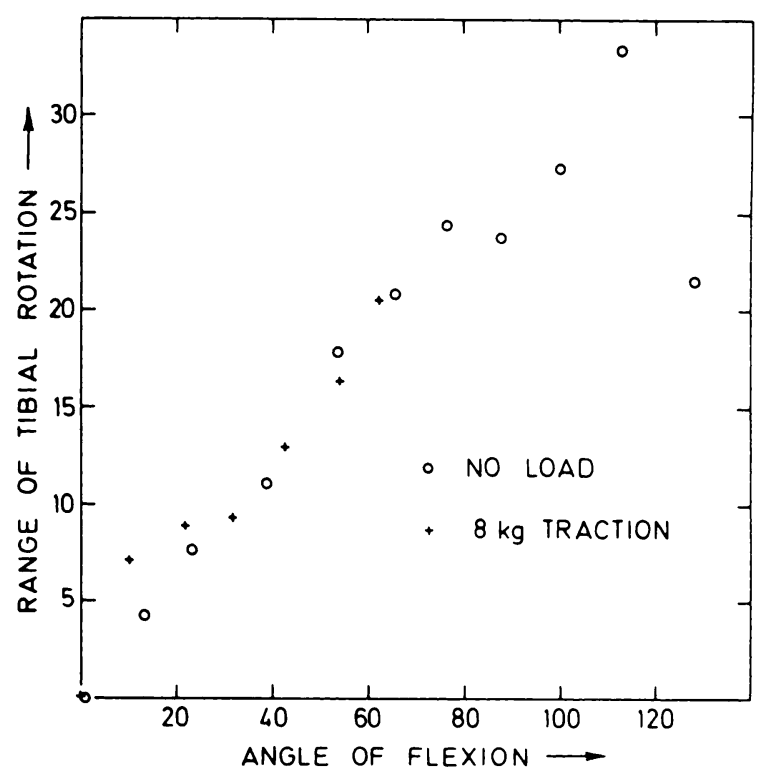

Fig. 11

Measured range of rotation of the tibia relative to the femur in a cadaveric knee under no load and under a traction force of 8 kilograms applied along the ankle-hip axis.

articular surfaces. If the tibial eminence is brought into contact, the components of the compressive forces in the plane of the tibial plateau could provide a couple to balance an applied torque. The lever arm of the couple arises from the relatively anterior position of one contact area and the posterior position of the other (Fig. 10).

We believe the contribution of the tibial eminence couple to be small. Deane (1970) has noted that the tibial plateau is tracked to allow the anteroposterior movement of the contact areas with relatively little distraction. Figure 11 shows the measured* range of tibial rotation of a cadaveric knee. The relative unimportance of the tibial eminence in resisting rotation may be deduced from the fact that the range of rotation remained much the same when a distracting force which separated the articular surface was applied. We will show (Fig. 22) that a similar range of tibial rotation was obtained when the tibial plateau was replaced by flat prosthetic surfaces which did not mimic the tibial eminence.

\section{Abduction and adduction with mediolateral translation.} A mediolateral force applied through the foot tends both to abduct and adduct the knee and to translate the bones relative to each other in the mediolateral direction. For a state of equilibrium to exist, an equal and opposite mediolateral force and a couple about the anteroposterior axis are transmitted across the joint (Fig. 12).

The collateral ligaments are the most advantageously disposed to resist abduction and adduction, in couple with compression on the contralateral condyles. The longest lever arms available are thereby employed. The collateral ligaments are not, however, the only 
structures which can passively resist such movements. The diagram suggests that tension in the cruciate ligaments can resist abduction or adduction though working through a shorter lever arm than do the collateral ligaments. We have found that each compartment of the knee has a degree of inherent stability independent of the other. If, in a cadaveric joint, one femoral condyle is excised, considerable stability is retained in both directions. The retained collateral and cruciate ligaments, acting albeit through much shorter patellar tendon can resist both abduction and adduction movement. In full extension, the tight posterior capsule exerts a similar effect for the same reasons. We test for the passive stabilising effect of the collateral ligaments with the knee slightly flexed. If this clinical test were done with the knee fully extended, the stability engendered by the tight posterior capsule would mask any deficiency in the collateral ligaments.

It is easy to show from statics (Fig. 12c) that the tensile and compressive forces needed to resist abduc-

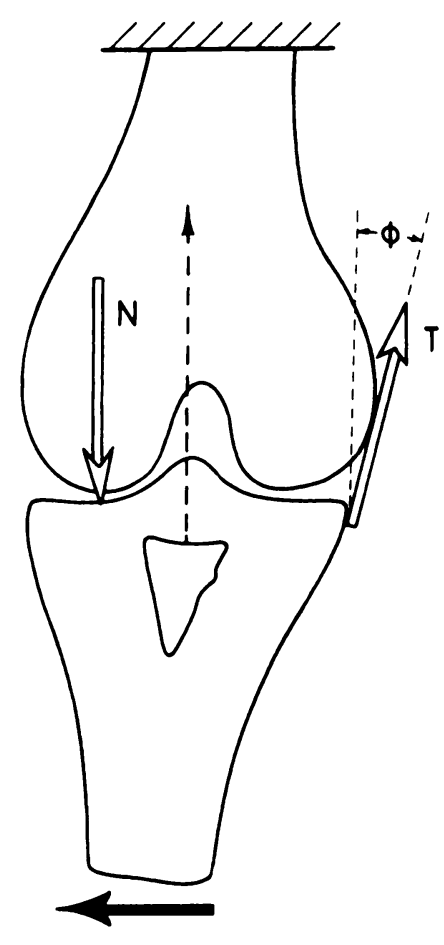

(a)

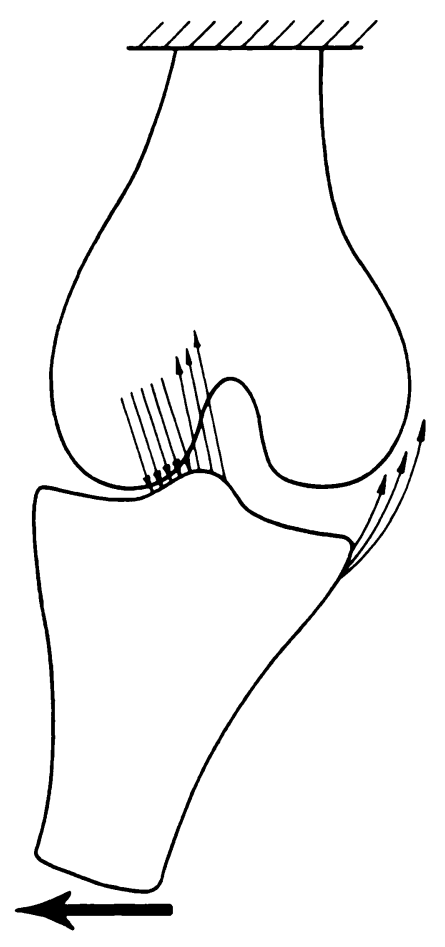

(b)

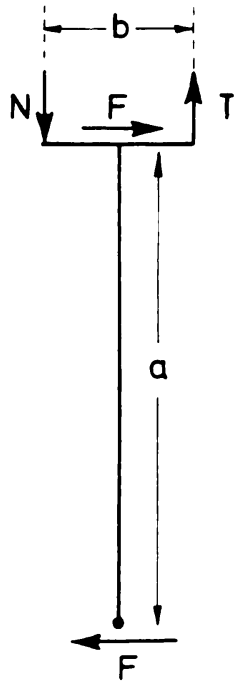

(c)

Fig. 12

(a) Simple couple resisting abduction or adduction. Dotted tension force represents active contribution of, for instance, patellar tendon force. (b) Abduction or adduction is usually accompanied by mediolateral translation which is resisted in part by the mediolateral component of the contact force. (c) Simple analysis of the tibia gives $N=T=F \frac{a}{b}$ where $\frac{a}{b}$ is approximately equal to 6 .

lever arms than in the intact joint, nevertheless effectively resist angulation.

At first sight, it might appear that ligamentous mechanisms are the only ones available to resist abduction or adduction at the knee, and that the joint's stability about this axis must, unlike other modes of stability, be the same during function as it is under minimal load. But, although there are no muscles able to induce abduction or adduction, it by no means follows that there are none capable of resisting such movements. Reference to Figure 12a will show that if the fulcrum of an adduction movement is within the contact area of the medial femoral condyle, all tension-bearing structures that span the joint lateral to that fulcrum contribute to the couple that resists adduction. Thus tension in the tion and adduction must be several times larger than the value of the mediolateral force applied. The lever arm available along the tibia to the applied force is much larger than that available to the joint forces. If the mechanism of Figure 3 were to operate alone to resist mediolateral translation, we estimate that an inclination of the soft tissues forces of about 10 degrees (the angle $\phi$ in Figure 12a) would be needed to balance the applied force. Such an inclination would imply mediolateral translation of the bones of about 5 millimetres, rather more than is observed. The joint is much more congruous when viewed from the front; the contact force could be transmitted, in part, by the tibial eminence and the mediolateral component of that force could help to balance the applied load. 
The function of the menisci. Fairbank (1948) suggested that the menisci transmit load between the femur and the tibia, and several recent investigations have confirmed the truth of his opinion (Seedholm, Dowson and Wright 1974; Shrive 1974; Walker and Erkman 1975; Krause et al. 1976). Estimates vary, but all are agreed that at least 50 per cent of the compressive force between the bones is carried by the menisci. They are relatively free to distort and can be displaced forwards and backwards upon the tibial condyles in order to maintain contact with the femur in all positions (Kapandji 1970). Such movements have been observed by arthroscopy to occur in the living during flexion, extension and rotation of the joint (Henry 1976).

The menisci act as conforming mobile bearing pads which spread the load transmitted between the femur and the tibia, increasing by a factor of about two the area of their contact, and thereby reducing the average pressure on the articulating surfaces.

\section{Résumé}

We have accounted for the control of all possible relative movements between the tibia and the femur, and described the transmission of all possible loads across the joint in terms of articular surface compression and soft tissue tension. The ability of the joint to transmit all possible loads and resist all possible movements derives solely from the ability of the articular surfaces to resist interpenetration and of the soft tissues to resist distraction.

The normal joint invites analogy with a wellpitched tent, which resists all forces tending to distort it by the development of tension in its guy-ropes and compression in its pole. It is the function of the articular surfaces to keep the bones apart; it is the function of the soft tissues to keep the bones together. The compressive stress is always at right angles to the articular surface, and the juxta-articular bone is therefore subject to this stress and designed to withstand it.

These generalisations are true of all synovial joints; they vary only in the proportion in which the soft and hard tissues contribute to stability. Instability results as readily from failure of an articular surface as it does from lengthening or rupture of a ligament, and in both cases the load-bearing function of the joint is prejudiced.

In offering this general description of the mechanics of the knee, we have not reported estimates of the magnitude of the forces in the various structures nor even, in any detail, specified the particular tensioncarrying elements involved in the transmission of any particular component of load. Such calculations may be prone to considerable error. Smith (1975) has shown that small errors in the estimates of the lengths of the lever arms can lead to large errors in the estimates of the forces. The joint, in mechanical terms, is a highly indeterminate structure. Crowningshield, Pope and Johnson (1976) had to use a model consisting of thirteen soft tissue elements to study the passive stability of the joint; were the features which contribute to active stability to be added, the calculation would be still more complex. Fortunately, to design a condylar replacement prosthesis, it is not necessary to know the precise distribution of stress throughout the soft tissues so long as it is realised that the ligaments confer passive stability by setting limits to the range of possible positions which the bones may adopt and that, within that range, the forces of gravity, of ground reaction and of muscle action determine their precise disposition. For these mechanisms to work, it is necessary that the articular surfaces, of themselves, should not resist any relative movement of the bones other than their interpenetration.

\section{THE DESIGN OF A PROSTHESIS}

The foregoing suggests some principles to guide the design of prostheses which replace diseased or worn articular surfaces while retaining the ligaments and muscles.

The components should be shaped to allow distracting, sliding and rolling movements between the bones; the components should apply only compressive stress to the juxta-articular bone; all surviving soft tissues should be kept and restored to their natural tensions; and the areas of contact between the prosthetic surfaces should be large enough to maintain the pressure under load at a level which the prosthetic materials can withstand.

It is not possible to attempt the design of prosthetic articular surfaces without immediately encountering a dilemma. If the components are to bear their loads through large areas of contact, they must fit one another in all postures of the joint - and the only shapes which will do so are spheres in spherical sockets. If two such devices are employed, one on each side of the joint, there can be but one axis of movement, a circumstance which, as we have seen, the ligaments are not designed to allow, and one which may rob the joint of certain valuable mechanical advantages. Such a joint is kinematically indistinguishable from a simple hinge with a transverse axle and rotation can only occur by dislocation of its surfaces. By their shapes, the tibial elements in this design resist rotation, anteroposterior and mediolateral translation and must therefore transmit the associated forces and couples. Rocking moments are thus engendered, and it would not be surprising if the elements in such a design were to come loose. All prostheses employing interlocked surfaces transmit shear and tension stresses to the bones, as demonstrated, for example, in Figure 13.

However, if we reject the ball-and-socket principle in favour of two articular surfaces which do not match, we necessarily transgress the fourth principle above. Figure 14 is the archetype of such designs. It may be ideal kinematically, allowing the convex femoral com- 
ponents to roll, slide and spin upon the tibia as the ligaments ordain, and its components (if friction between them is ignored) transmit largely compressive stress to the bones beneath the $\mathrm{m}$. But the contact areas are small, and the pressures to be sustained by the materials are therefore high, with the likelihood of excessive creep and wear.

Many current designs seek compromise solutions to the dilemma; their components are made so that the opposed contours almost match, but not quite. Such
There is one way of avoiding the need to compromise at all which is similar to the natural solution. Figures 15,16 and 17 show the same model as in Figures 5 to 7 but with the addition of a closely fitting but unconstrained washer trapped, only by its shape, between the two articular surfaces. A perfect fit with good load-bearing can be achieved without altering the kinematics of the model at all. The function of the natural menisci is similar to that served by the washer in the model, though the mechanism of load-transmission

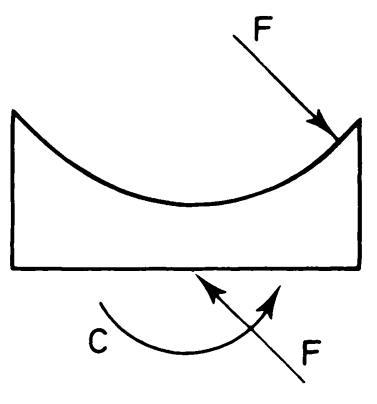

(a)

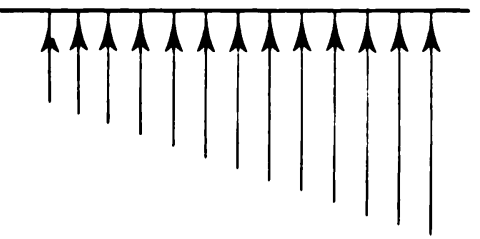

(b)

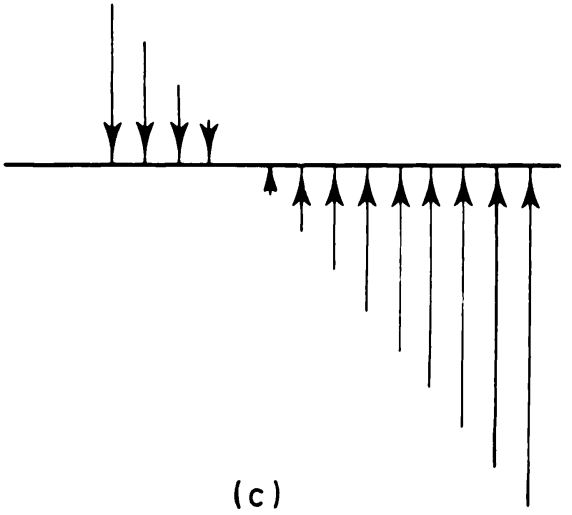

Fig. 13

(a) Contact force on a socket-like tibial component is balanced by an equal and opposite force and a couple. (b) The normal stress applied to the juxta-articular bone is entirely compressive if the couple is small but (c) can be tensile over part of the interface if the couple is large.

compromises represent an acceptance of some of the disadvantages of incongruity of the components to gain some of the advantages of unconstrained movement. The large variety of such designs differ from each other

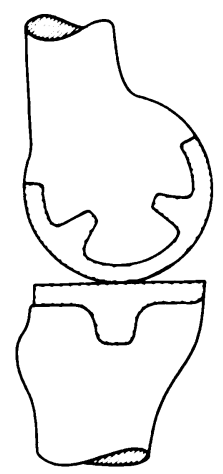

Fig. 14

Convex femoral components on flat tibial components.

mainly in the fine detail of the surface geometry considered necessary to complement the function of the soft tissues. Accuracy of implantation then becomes critical to the stability of the resulting arthroplasty. within the menisci is quite different. In the model, a rigid and undeformable washer transmits load in compression, while the natural meniscus transmits compressive force by the development of tension in its collagen fibres which are mainly aligned with its circumference (Bullough et al. 1970).

Figures 18 and 19 show a cadaveric knee into which a prosthesis has been implanted. The femoral components are spherical; the tibial components are flat. These elements were cemented to the bone ends. Between the $m$ lie plastic washers, each spherically concave above and flat below, exactly fitting the shapes of the metal components. The washers were made in several thicknesses (in 1 millimetre steps) and, from this range, two were chosen which "snapped" into place, rendering the ligaments tight. This prosthesis has been implanted into twenty-five cadaveric joints. Femoral components of the same size (radius 24 millimetres) were used in all. The femoral elements were so adjusted on the bone, by appropriate cuts, that the gaps existing between their surfaces and those of the tibial elements were the same at full extension as they were at 90 degrees of flexion. The washers filled those gaps and were trapped in position because of their shape.

Flexion and extension. Comparison of Figures 18 and 19 shows that, as the joint flexes and extends, the washers move backwards and forwards upon the tibial flats, as 


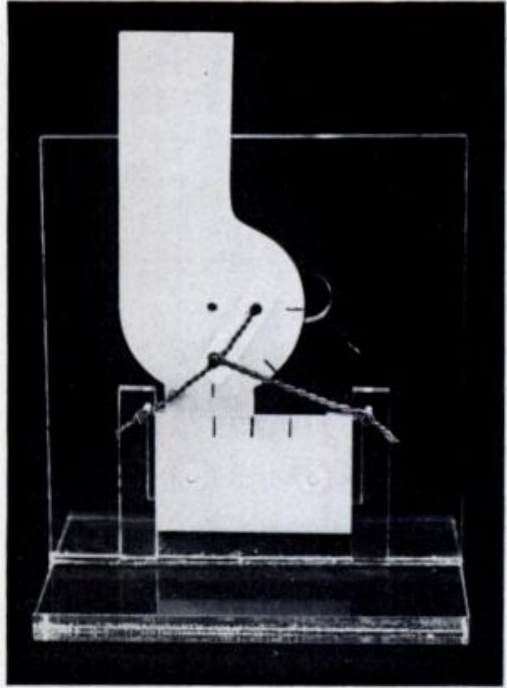

Fig. 15

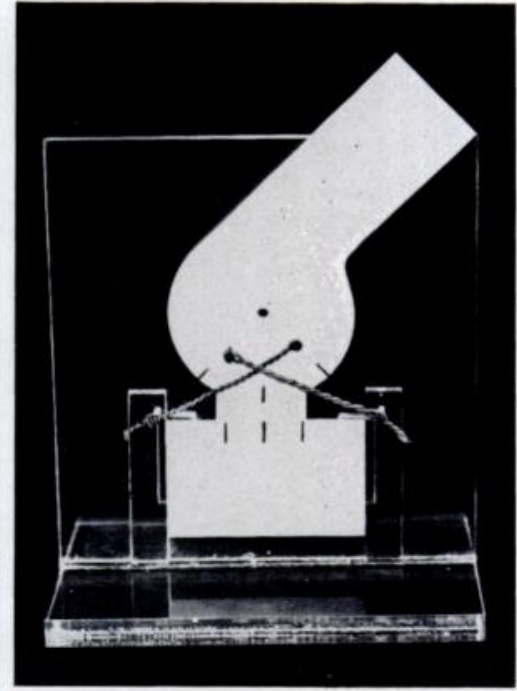

Fig. 16

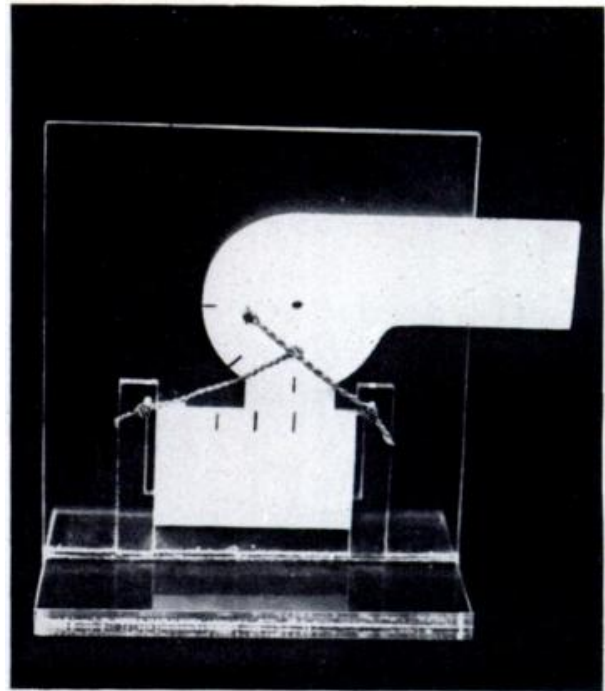

Fig. 17

Figures 15, 16 and 17-Two-dimensional model with a meniscal "washer". Movement of femur relative to tibia is the same as in Figures 5 to 7 but the contact areas are larger.

did the washer in the two-dimensional model. This movement of the washers was found to be obligatory, for if it was artificially blocked, the joint "locked" and would neither flex nor extend. The excursion of the washers was between 0.6 and 1.2 centimetres during flexion to 90 degrees.

In those cadaveric joints that initially enjoyed a full range of movement this was reproduced after implantation of the device. Beyond about 120 degrees of flexion, the backwards excursion of the washers brought their posterior edges to the posterior limit of the tibial plateaux and sometimes beyond, but posterior dislocation never occurred.

Rotation. Comparison of Figures 20 and 21 shows that medial and lateral rotations were allowed by reciprocal

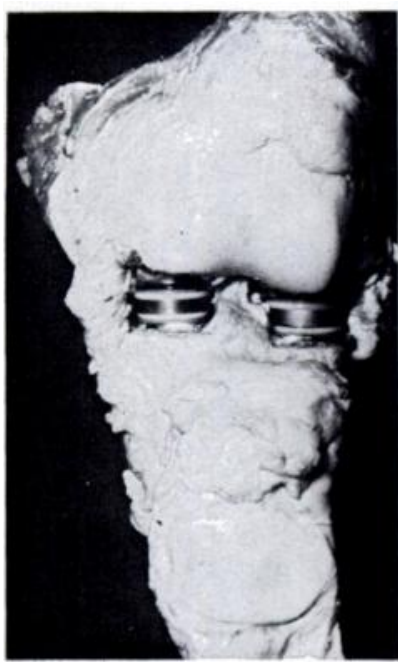

Fig. 18

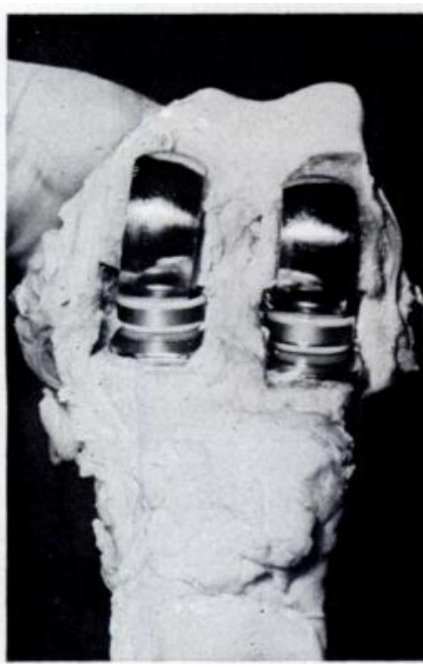

Fig. 19 backward and forward movements of the femoral condyles upon the tibia, a movement accommodated by the washers sliding backwards and forwards upon the tibial flats.

In Figure 22, the range of rotation available at various positions of flexion in an intact cadaveric knee is compared with the same joint after implantation of the prosthesis. The range of rotation was determined with the joint unloaded, and in both cases the measured range was similar to that of Markolf, Mensch and Amstutz (1976).

Stability. The most striking feature of the function of the implant was the degree of passive stability which it could confer and the fine control which choice of washer thickness exercised. An appropriate choice gave com-

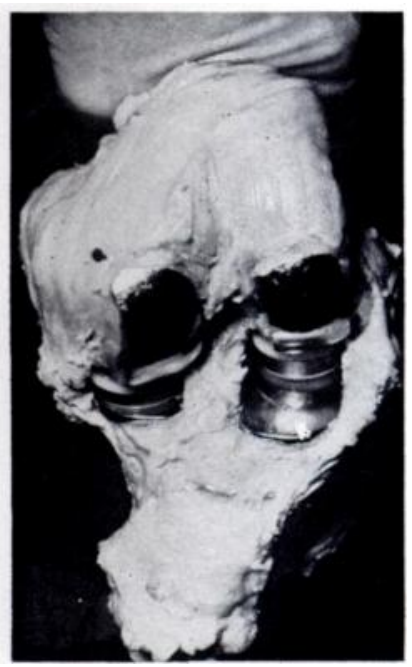

Fig. 20

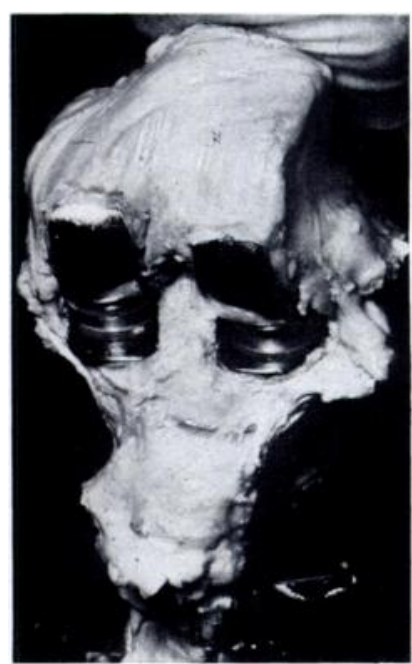

Fig. 21
The "meniscal" prosthesis demonstrating the obligatory anteroposterior movement of the washers. Figure 18-In extension. Figure 19-In flexion.
Rotation of the tibia, demonstrating the reciprocal movement of the washers. Figure 20-Lateral rotation. Figure 21-Medial rotation. 
plete lateral stability throughout the range of flexion; extension was accurately limited and the joint locked in full extension as in the normal knee, allowing no rotation in that position (Fig. 22). The drawer test, performed with the knee flexed, demonstrated a normal range of anteroposterior glide of the femoral condyles and their washers upon the tibial flats.

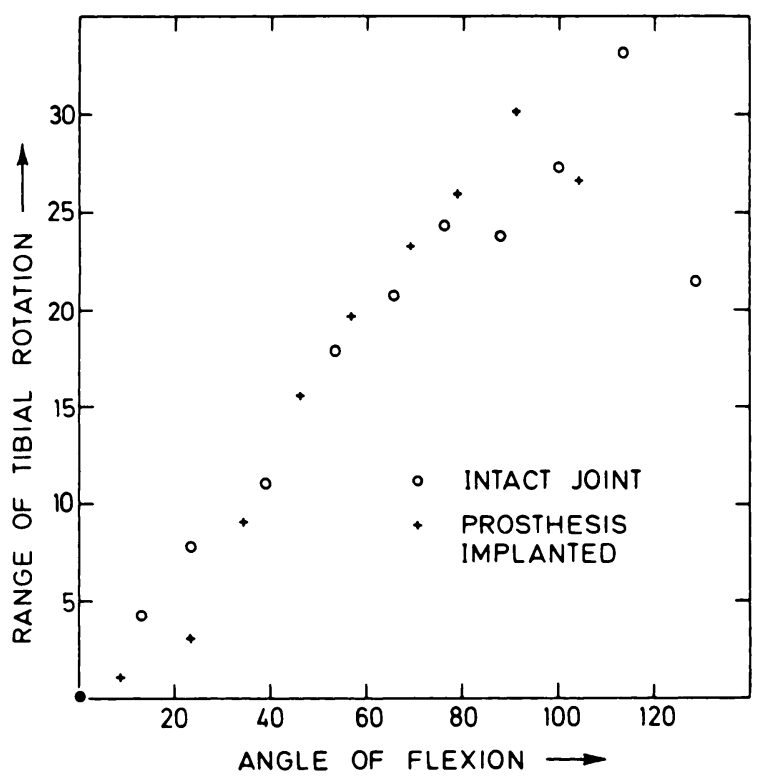

Fig. 22

Measured range of tibial rotation of a cadaveric knee under no load before and after implantation of the "meniscal" prosthesis.

All these features of passive stability, depending as they do on the development of tension in the ligaments, could be diminished or enhanced by reducing or increasing the thickness of the washers. Reproduction of normal stability required an accuracy to within 1 to 2 millimetres.

Effects of division of the cruciate ligaments. In some cadaveric joints, one and then both the cruciate ligaments were divided after implantation of the prosthesis. The effect was always to render the joint less stable. Backward and forward movements of the washers still occurred with rotation and flexion or extension, but with much less precision. The drawer test became positive, the range of rotation increased, and stability in abduction and adduction diminished. These changes occurred because the washers, which had hitherto been of adequate thickness to tension the ligaments, were now obviously too thin.

As thicker washers, in millimetre steps, were inserted, the lateral stability of the joint was gradually regained and its rotary freedom diminished to near normal again. The control of the anteroposterior location of the washers on the tibial flats also improved, but never achieved the precision of a joint with intact cruciate ligaments.

\section{Clinical application}

Having shown that the prosthesis was capable of restoring passive stability and natural mobility in the cadaveric knee, and that the unconstrained washers did not displace, some implants have been undertaken in patients suffering from advanced rheumatoid arthritis or osteoarthritis. In due course the results of the clinical trial will be reported in full. It is already possible to state that, in joints rendered grossly unstable by disease, stability can be re-established by the use of this prosthesis almost as readily at operation as in the laboratory.

In the two disease processes mentioned, instability was found to result mainly from erosion of the articular surfaces. Once the surfaces had been replaced, the remaining ligaments proved capable of resuming their former function. In some cases, the anterior cruciate ligament was absent, but we have not yet encountered a joint which lacked both cruciates.

In the living, we have been able to observe the additional effects of muscle force, ground reaction and gravity upon the passive stability provided by the tightened ligaments. The recovery of active stability has been the most remarkable feature in the small series of patients operated upon.

\section{DISCUSSION}

In our mechanical analysis of the human knee, we concluded that it was the function of the articular surfaces to hold the bones apart. The prosthesis just described does that-and nothing else. Its components can offer no significant resistance to shear or tension stresses, and all loads must be transmitted through the reconstructed knee, as they are through the natural joint, by a combination of pressure normal to its articulating surfaces and tension in the soft tissues. The results of the experiments just described confirm that, if the prosthesis fulfils this one function, the retained soft tissues can faithfully reproduce the freedoms and limitations of movement seen in the natural joint. But the soft tissues can only do so if the "spacer effect" of the prosthesis accurately adjusts their tension and maintains it throughout the range of movement. This it can do only if its design imposes no arbitrary axes upon such movements.

In addition to the clear advantage of spreading the load, the use of interposed washers confers upon the design an important advantage, that the tension in the ligaments can be adjusted after the metal components have been fixed to the bones.

Since it may be significant in its practical application, we draw attention to the behaviour of the prosthesis in those cadaveric joints in which the cruciate ligaments were divided. The experiment confirms the commanding role the cruciates play in the control of the intact joint, but it also demonstrates that the other soft 
tissues, designed as they are to accommodate the natural combination of rolling and sliding movements, can themselves command such movements if the cruciates are not functioning. They command such movements only if they are rendered tight enough to do so by implanting thicker washers than the intact cruciates will allow.

The experiments also confirm that the polyaxial movements of the human knee are the result of the crossed form of its ligaments and not a direct consequence of the polycentric curvatures of the femoral condyles. It is at first difficult to understand how a circular surface on a flat one can keep the ligamentous structures at a lifelike tension throughout the range of movements. That they can do so is shown by the fact that all aspects of passive stability were reproduced, as evidenced, for example, by the similarity of the two graphs in Figure 22. It appears that the significance of the generally helical shape of the femoral condyles has been wrongly over-emphasised. The explanation of the fact that a crossed system of ligaments can accommodate an infinite number of pairs of surfaces provided that they are free to roll and slide upon one another is given in some detail in the Appendix to this paper.

The question whether the inclusion of unconstrained washers within a prosthesis will prove a practical as well as a theoretical solution to the design dilemma referred to cannot yet be answered. In twenty-five cadaveric joints, tested in a rig which allowed normal knee movements, the washers did not dislocate.

If a washer of the right size is inserted in the first instance, it could dislocate only if the gap between the fixed components subsequently were to open. This could happen if the ligaments were to stretch or if one or other fixed component were to sink into the bone.

No form of attachment has been used to hold the washers in place other than the entrapment provided by their geometry. The retained soft tissues, by resisting distraction of the joint, resist dislocation of the prosthesis just as they resist dislocation of the normal joint. Indeed, were there to be any other restraint in the form of tracks or stops to limit the freedom of the washers to translate within the range defined by the ligaments, such restraints would inevitably cause the fixed components to transmit shear and tension stresses to the bone. Experiments with "semi-constrained" washers showed that constraints which operated within the range of the ligaments did indeed result in rocking of the tibial components. If such tracks or stops only came into operation outside the range normally allowed by the ligaments, they could give an extra security against dislocation when the joint is unloaded and its surfaces liable to distract.

Will the soft tissues of diseased joints maintain for long the function demanded of them in this design? We have shown that they can reassume initial control and we rely on the general observation that biological materials thrive when functioning within their design range. If, however, this hope is not realised, then the prospects for all condylar replacement prostheses must be poor. For, if the tensile components of load are not wholly borne by the soft tissues, then the prosthetic surfaces cannot escape transmitting shear and tension stress to the bones to which they are attached. Any form of interlocking of the prosthetic surfaces must have this effect. Our mechanical analysis suggests that juxta-articular bone may fail when stressed in this way, and it explains why constrained designs of prostheses may require intramedullary stems to carry the forces across the intracapsular bone to the shaft, the tubulated form of which is designed to resist all components of load.

The problems of wear and creep common to all joint prostheses are limited in this design to the washers. Laboratory tests show that creep in the plastic washer can be much reduced by placing a metal ring around its circumference (Figs. 18 to 21 ). By preventing radial expansion, in the manner of a tyre on a farmyard cartwheel, the ring reduces the maximum shear stress in the washer and, therefore, its propensity to flow. Wear rates, deduced from the data of Dowson, Atkinson and Brown (1975), can be expected to be small.

At the inception of these studies, Dr N. G. Shrive demonstrated the weight-bearing role of the menisci (Shrive, N. G. (1974) D.Phil. Thesis, University of Oxford) and was involved in the preliminary discussions which led to the design of the "meniscal" prosthesis (Goodfellow. J. W., O'Connor, J. J., and Shrive, N. G. (1974). British Patent Application No. 49794/74).

APPENDIX:

GEOMETRY OF CONDYLAR SURFACES

The kinematics of the two-dimensional model (Figs. 5 to 7) can be analysed to demonstrate the characteristics of crossed ligament designs and the surface shapes they can accommodate.

The instant centre of the femur relative to the tibia, the point about which either bone may be considered to be spinning relative to the other, is always located at the point at which the two ligaments cross. As the joint flexes and extends, the point of cross-over moves relative to both bones. The paths of the instant centre, on both bones, may be determined by finding the location of the cross-over point relative to each bone for several positions of the joint. These paths consist of pairs of points, one attached to each bone, each pair coinciding for one position of the joint and, when coincident, moving with exactly the same velocity. The two paths therefore represent the shapes of those surfaces, one attached to each bone, which would complement the ligament design and roll without slip on each other. The curves are called the centrodes of the mechanism. For a given mechanism the centrodes are unique.

Three sets of centrodes are shown in Figure 23 for three different designs. The femoral centrode in each case is closely circular but the tibial centrode is concave upward, concave downward or flat, depending on whether the femoral attachment distance is less than. greater than or equal to half the tibial attachment distance. In the latter case, the design is called the Chebychev straight-line mechanism (Hall 1961), used to roll a circular surface upon a flat surface without slip. 
The measured dimensions ${ }^{*}$ of the cruciate ligaments in the human knee ( $\mathrm{AB}=22$ millimetres, $\mathrm{CD}=12$ millimetres) approximate to those of the mechanism 2 in the figure, and so the centrodes of the human cruciates are approximately a circle on a flat.

A circle of relatively smaller or relatively larger radius than that of the centrode can be used, but in both instances some sliding movements between the surfaces are introduced. If a circle of radius less than that of the centrode is employed, the sliding will be in the same direction as the rolling movement. If a larger radius is used, the sliding and rolling occur in opposite directions. For the surfaces to make contact at about the level of attachment of the cruciates to the tibia, a circle of larger diameter than that of the femoral centrode is required, and such a surface will roll backwards while sliding forwards, and vice versa (Figs. 5 to 7 ).

The number of pairs of surfaces which a crossed four-bar linkage can accommodate is, in fact, infinite. The shape of one surface having been chosen quite arbitrarily, it is possible by simple geometry to deduce the shape of the only surface which will complement it, and each pair of surfaces will exhibit a particular combination of rolling and sliding movements. We deduce that the articular surfaces of the knee form one of many possible combinations which could complement its ligaments. The circle on the flat is a good approximation to another.

Since circles of differing radii can all be accommodated by a ligament design of fixed dimensions, it follows that a circle of fixed radius can accommodate ligaments of varied dimensions. If the ligaments of large and small human knees vary only in their absolute and not in their proportional dimensions, a circular femoral prosthetic surface of one radius can accommodate them all, and only the relationship of the level of the articulation to the cross-over point of the cruciates need vary. This is the theoretical explanation for the observations reported in this paper that, in forty-one human knees, femoral components of radius 24 millimetres accommodated the ligaments in every case, the level of the articulation varying as did the quantity of bone removed from the tibia and the thickness of the interposed washers.

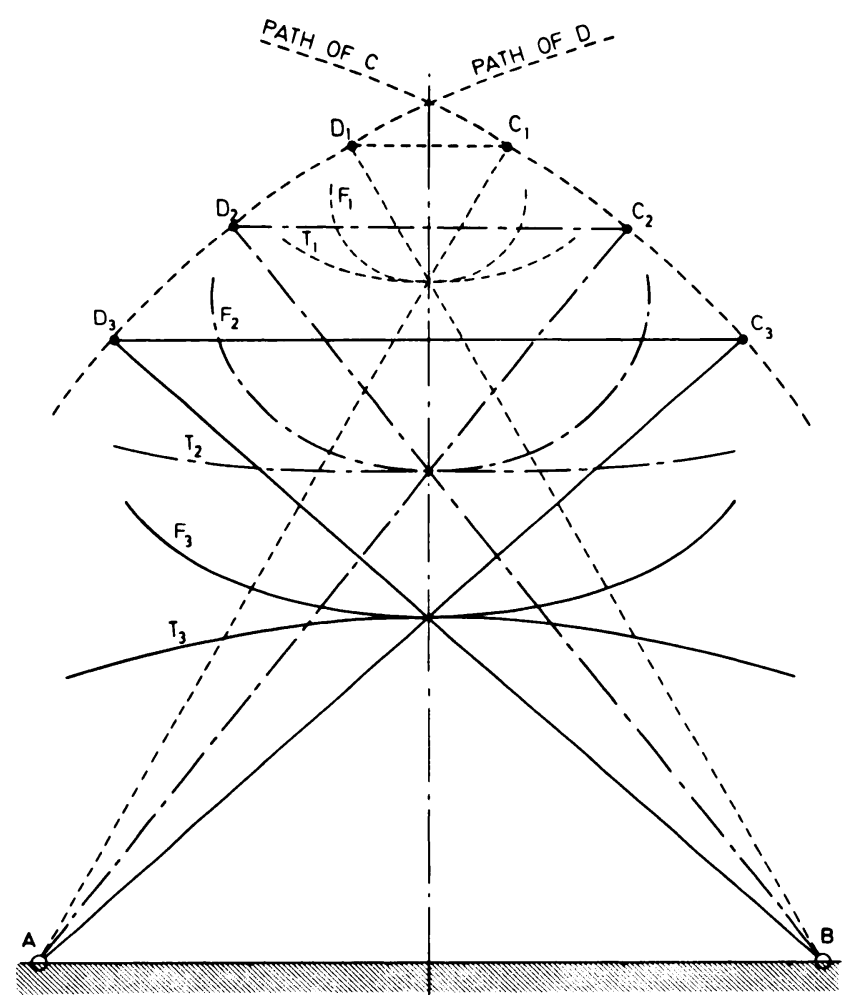

Fig. 23

Loci of the instantaneous centre (centrodes) relative to the femur $\left(F_{1}\right.$, $F_{2}, F_{3}$, ) and relative to the tibia $\left(T_{1}, T_{2}, T_{3}\right)$ for three designs of cruciate mechanism having femoral attachment distances $\left(C_{1} D_{1}, C_{2} D_{2}, C_{3} D_{3}\right)$ equal to $0.2,0.5$ and 0.8 of the tibial attachment distance $A B$. Within a certain range of flexion, the femoral centrodes are circular. Tibial centrode $T_{2}$ is flat within a flexion range of about $60^{\circ}$.

*Although Kapandji (1970) states that posterior cruciate is three-fifths the length of the anterior, when viewed from the side they appear much more nearly equal in length.

\section{REFERENCES}

Bullough, P. G., Munuera, L., Murphy, J., and Weinstein, A. M. (1970) The strength of the menisci of the knee as it relates to their fine structure. Journal of Bone and Joint Surgery, 52-B, 564-570.

Bourne, R., Goodfellow, J. W., and O'Connor, J. J. (1978) A functional analysis of various knee arthroplasties. Proceedings of the 24th Meeting. Orthopaedic Research Society, Dallas.

Crowningshield, R., Pope, M. H., and Johnson, R. J. (1976) An analytical model of the knee. Journal of Biomechanics, 9, $397-405$.

Deane, G. (1970) Contact Print Studies in the Human Knee Joint. M.Sc. Thesis, University of Surrey.

Dowson, D., Atkinson, J. R., and Brown, K. (1975) The wear of high molecular weight polyethylene with particular reference to its use in artificial human joints. Advances in Polymer Friction and Wear, 5B, 533.

Fairbank, T. J. (1948) Knee joint changes after meniscectomy. Journal of Bone and Joint Surgery, 30-B, 664-670.

Hall, A. S. (1961) Kinematics and Linkage Design. Englewood Cliffs: Prentice-Hall.

Henry, A. (1976) Personal Communication.

Huson, A. (1974) The functional anatomy of the knee joint: the closed kinematic chain as a model of the knee joint. In The Knee Joint, pp. 163-168. International Congress Series No. 324. Amsterdam: Excerpta Medica.

Kapandji, I. A. (1970) The Physiology of the Joints. Volume 2. Lower Limb, p. 120. London and Edinburgh: Churchill Livingstone.

Krause, W. R., Pope, M. H., Johnson, R. J., and Wilder, D. G. (1976) Mechanical changes in the knee after meniscectomy. Journal of Bone and Joint Surgery, 58-A, 599-604.

Markolf, K. L., Mensch, J. S., and Amstutz, H. C. (1976) Stiffness and laxity of the knee-the contributions of the supporting structure. A quantitative in vitro study. Journal of Bone and Joint Surgery, 58-A, 583.

Seedhom, B. B., Dowson, D., and Wright, V. (1974) The load-bearing function of the menisci: a preliminary study. In The Knee Joint, pp. 37-42. International Congress Series No. 324. Amsterdam: Excerpta Medica.

Shrive, N. (1974) The weight-bearing role of the menisci of the knee. Journal of Bone and Joint Surgery, 56-B, 381.

Smith, A. J. (1975) Estimates of muscle and joint forces at the knee and ankle during a jumping activity. Journal of Human Movement Studies, $1,78-86$.

Walker, P. S., and Erkman, M. J. (1975) The role of the menisci in force transmission across the knee. Clinical Orthopaedics and Related Research, 109, 184-192. 